\title{
0 megaesôfago tratado cirurgicamente: perfil epidemiológico dos pacientes operados no Hospital de Clínicas da Universidade Estadual de Campinas entre 1989 e 2005
}

\author{
Surgically treated megaesophagus: epidemiological profile of \\ patients operated in the Clinical Hospital of the State \\ University of Campinas between 1989 and 2005 \\ Gustavo Carvalho de Oliveira ${ }^{1}$, Luiz Roberto Lopes $^{1}$, Nelson Adami Andreollo ${ }^{1}$ \\ e João de Souza Coelho Neto ${ }^{1}$
}

\begin{abstract}
RESUMO
0 megaesôfago é uma das manifestações da doença de Chagas, cujo tratamento cirúrgico ó o que apresenta melhores resultados. Neste estudo retrospectivo, avaliou-se o perfil epidemiológico dos pacientes operados no Hospital de Clínicas da Universidade de Campinas entre 1989 e 2005 , quanto a: naturalidade e procedência, provável local de contágio, idade, grau do megaesôfago, etiologia, duração da disfagia e sua evolução, outras doenças associadas e modalidade cirúrgica adotada. 0 método foi a análise de 390 prontuários desses doentes, junto ao Serviço de Arquivo Médico do Hospital de Clínicas da Universidade de Campinas. Os resultados permitiram estabelecer: as regiões endêmicas dos pacientes chagásicos atendidos nesse Serviço, a naturalidade/procedência e a caracterização do grupo. Após análise detalhada, foram obtidas a média de idade = 47 anos, e a duração média da disfagia = 9,47 anos. Observa-se que: a) em 84,4\% dos pacientes a disfagia instalou-se progressivamente; b) 306 (78,5\%) pacientes apresentaram etiologia chagásica; c) em 48\% deles, houve prevalência do grau 2 (48\%); d) 89,8\% dos pacientes foram submetidos à cardiomiotomia; e) houve associações freqüentes a gastrites, esofagites, megacólon, hipertensão arterial e cardiopatias.
\end{abstract}

Palavras-chaves: Megaesôfago. Doença de chagas. Cardiomiotomia. Acalásia idiopática do esôfago. Esôfago.

\begin{abstract}
Megaesophagus is one of the manifestations of Chagas disease and surgical treatment is the approach that presents the best results. In this retrospective study, the epidemiological profile of patients operated in the Clinical Hospital of University of Campinas between 1989 and 2005 was evaluated with regard to: place of birth, place of residence, probable place of infection, age, degree of megaesophagus, etiology, duration and evolution of dysphagia, other diseases in association and the type of surgery chosen. The method used was to analyze the 390 medical files of these patients, at the hospital's medical archive service. The results made it possible to establish the endemic regions, place of birth and place of residence of the patients with Chagas disease attended at our clinic, and to characterize the group. After detailed analysis, it was found that the mean age was 47 years and the mean duration of dysphagia was 9.47 years. It was observed that: a) in $84.4 \%$ of the patients, dysphagia took hold progressively; b) 306 (78.5\%) patients presented Chagas disease etiology; c) grade 2 was prevalent in $48 \%$; d) $89.8 \%$ of the patients underwent cardiomyotomy; and e) there were frequent associations with gastritis, esophagitis, megacolon, arterial hypertension and cardiopathy.
\end{abstract}

Key-words: Megaesophagus. Chagas disease. Cardiomyotomy. Idiopathic achalasia of the esophagus. Esophagus.

A tripanossomíase americana descrita por Carlos Chagas em 1909 constitui, ainda hoje, um problema sócio-econômico nacional e regional que aflige, principalmente, os países do cone sul da América, prevalentemente. 0 nível endêmico decorreu, principalmente, devido à domiciliação dos vetores motivada por más condições de habitação, esgotamento de fontes alimentares originais (ação antrópica destrutiva) e características dos vetores (fototropismo e hematofagia) ${ }^{13}$.

O Banco Mundial (World Development Report, 1993) estudou o peso relativo da doença de Chagas, comparado ao de outras enfermidades transmissíveis endêmicas na América Latina e Caribe, e avaliou os pacientes chagásicos quanto aos anos de

\footnotetext{
1. Faculdade de Ciências Médicas, Departamento de Cirurgia. Universidade Estadual de Campinas, Campinas, SP. Apoio financeiro: Fundação de Amparo à Pesquisa do Estado de São Paulo (FAPESP), processo nº 06/50122-1.

Endereço para correspondência: Dr. Gustavo Carvalho de Oliveira. R. Shigeo Mori 1460, Cidade Universitária, 13083-765 Campinas, SP. Tel: 551992252134

e-mail: gustavok@fcm.unicamp.br

Recebido em: 29/10/07

Aceito em: 10//03/08
} 
vida ajustados à incapacidade". Na ocasião, concluiu que nestes aspectos, a doença de Chagas só era superada pelo conjunto de enfermidades diarréicas e respiratórias e pela AIDS. Ressalta-se, pois, a importância desse problema de saúde pública ${ }^{13}$.

Parece haver um diferente tropismo de cepas de Trypanosoma cruzi, o que explica o fato de ser muito comum o diagnóstico de formas digestivas, na região central do Brasil, e a baixa prevalência de cardiopatias, no norte do Equador ${ }^{13}$.

No Brasil, o inquérito sorológico, realizado entre 1975 e 1980, estimou que as maiores incidências se davam em Minas Gerais e Goiás. Em 1979, ocorriam cerca de 100 mil novos casos anuais, com freqüentes infestações agudas.

Na década de 70, o Ministério da Saúde tomou a iniciativa de controlar a transmissão vetorial de Chagas; em 1980, iniciouse a borrifação com inseticida em áreas endêmicas. Desde então, o número de pacientes com forma aguda da doença foi drasticamente reduzido e desapareceu na maioria das regiões endêmicas. Isso sugere que houve uma transmissão vetorial 85\% menor, comparado à década anterior. Apesar disso, estima-se uma população de quase oito milhões de infectados, com uma média de 17.000 mortes anuais pela doença ${ }^{2}$ e $40 \%$ desses infectados apresentam megaesôfago ${ }^{4}$.

0 comprometimento do trato digestivo, na doença de Chagas, ocorre com freqüiência variável, entre 3,1\% e 13\%, conforme a região endêmica estudada. A esofagopatia chagásica precede, de modo geral, a cardiopatia chagásica, pelo menos em relação à exteriorização clínica Verificam-se muitos chagásicos portadores de megaesôfago, com dez ou mais anos de doença, sem cardiopatia evidente ${ }^{2}$.

0 megaesôfago é uma das formas clínicas do mal de Chagas que, embora de natureza benigna, tem caráter crônico e progressivo, o que determina repercussões relevantes sobre o estado nutricional e psíquico dos pacientes. Ele pode ser diagnosticado em qualquer idade, mas é mais frequiente entre 20 e 40 anos e predomina no sexo masculino ${ }^{5}$. A destruição e a ausência de plexos nervosos intramurais de Auerbach do esôfago determinam a ausência de peristaltismo ao nível do corpo do órgão e a não abertura de seu esfíncter inferior (acalásia), em resposta à deglutição. Ocorre, em consequiência, estase esofágica e, inicialmente, incoordenação motora, dilatação e diminuição da capacidade de contração do esôfago ${ }^{5}$.

A doença de Chagas é o único fator etiológico realmente comprovado de megaesôfago, embora se admita que algumas drogas, como a iperite, possam determinar lesão plexular e o aparecimento da doença. A dilatação congênita já foi descrita em várias raças de cães, mas a comprovação de megaesôfago hereditário em humanos é prejudicada devido à sua raridade 5 .

A disfagia é a manifestação que leva o doente a procurar 0 médico e determina o diagnóstico. Tem evolução longa, de vários anos, e é progressiva. Inicialmente, ocorre quando da ingestão de sólidos, depois para ingestão de pastosos, chegando a ocorrer inclusive com ingestão de líquidos. A regurgitação, sem ocorrência de náusea, caracteriza o megaesôfago ${ }^{5}$. Esclarecidas as suspeitas clínica e epidemiológica, realizam-se exames para o diagnóstico diferencial. A radiografia contrastada do esôfago quase sempre confirma o diagnóstico de megaesôfago e a endoscopia pode ser classificada como exame complementar. Esse exame confirma a ausência de obstrução orgânica da cárdia e a presença possível de tumores despercebidos ao exame radiológico ${ }^{6}$.

Existem diversas classificações para o grau de megaesôfago, mas será adotada a de Mascarenhas ${ }^{11}$ que se baseia, fundamentalmente, no diâmetro transverso do órgão, a saber: grau I- até $4 \mathrm{~cm}$, grau II- de 4 a $7 \mathrm{~cm}$, grau III- 7 a $10 \mathrm{~cm}$, grau IV- mais de $10 \mathrm{~cm}^{11}$. Essa classificação é importante para adequar o tratamento, por procedimento cirúrgico, com resultados mais eficientes, menor morbidade e melhor qualidade de vida 3 .

0 tratamento clínico do megaesôfago é meramente paliativo; serve simplesmente para melhorar a sintomatologia do paciente que tem dificuldade em deglutir. Dieta líquida, lavagens por sonda, antiácidos e anti-espasmóticos também são usados para melhorar a sintomatologia. A dilatação da cárdia, atualmente feita com a sonda de Savary-Gilliard, de materiais flexíveis, utilizada com auxílio de um endoscópio, nos grau I e II pode ser de bom auxílio, pois a resistência do esfíncter é reduzida, o que proporciona maior esvaziamento do esôfago ${ }^{4}$.

Para casos mais avançados, utiliza-se o tratamento cirúrgico. Até o grau III, usa-se, classicamente, a cardiomiotomia de Heller modificada. Esse procedimento consiste na incisão longitudinal do esôfago e da parte proximal do estômago, de modo a dissecar a cárdia e manter a mucosa intacta. A fixação do fundo gástrico, de modo a funcionar como válvula anti-refluxo anterior foi a alteração descrita por Pinotti. Este é um procedimento amplamente utilizado no Hospital de Clínicas da Universidade Estadual de Campinas, pela disciplina de Moléstias do Aparelho Digestivo da Faculdade de Ciências Médicas. Em casos extremamente avançados (graus IV, principalmente), utiliza-se a esofagectomia (excisão total ou parcial do esôfago) com grau de morbidade considerável. Preferencialmente, é feita uma laparotomia, sem toracotomia. A reconstrução do trânsito do alimento deve ser realizada com o estômago (esofagogastroplastia), embora em algumas situações seja necessário o uso do cólon. A esofagogastroplastia apresenta a vantagem de necessitar de somente uma anastomose, enquanto se utilizar o cólon, três anastomoses são necessárias. Além disso, o uso do estômago neste procedimento implica um índice muito menor de infecçã $0^{4}$.

Diante disso, este trabalho tem por objetivo analisar o perfil epidemiológico dos pacientes operados de megaesôfago (chagásico ou idiopático) no Hospital de Clínicas da Unicamp entre 1989 e 2005. Os resultados advindos desse estudo são relevantes para analisar o perfil dos pacientes atendidos. A verificação da freqüiência da acalásia idiopática do esôfago neste Serviço é importante e pode estimular novos estudos sobre a acalásia idiopática do esôfago (AIE).

\section{PACIENTES E MÉTODOS}

Foi feito um levantamento retrospectivo, junto ao Serviço de Arquivo Médico (SAM) do Hospital de Clínicas da UNICAMP, de 390 prontuários dos pacientes operados por megaesôfago, a 
partir de 1989 até 2005. Mediante a elaboração de uma ficha/ questionário, os dados foram organizados conforme: idade, sexo, naturalidade, procedência, duração média da disfagia, sorologia para doença de Chagas (positiva ou negativa), outras doenças importantes e/ou associadas, grau do megaesôfago, intervenção cirúrgica adotada. 0 Comitê de Ética em Pesquisa da FCM/UNICAMP aprovou a realização do projeto (parecer $\left.\mathrm{n}^{0} 700 / 2005\right)$.

0 prontuário dos pacientes foi a fonte de dados principal e os métodos pressupostos para a verificação de dados específicos, como o grau do megaesôfago e a etiologia para doença de Chagas foram: diâmetro transverso do esôfago (por achados radiológicos), na classificação de Mascarenhas ${ }^{11}$, com: grau I- até $4 \mathrm{~cm}$, grau II- de 4 a $7 \mathrm{~cm}$, grau III- 7 a $10 \mathrm{~cm}$, grau IV- mais de $10 \mathrm{~cm}^{13}$, conforme descrito anteriormente. Exames com técnicas de imunofluorescência com duas sorologias negativas, antecedentes epidemiológicos negativos para a doença de Chagas e ausência de cardiopatia e megacólon associados qualificam a acalasia como idiopática, enquanto uma sorologia positiva aponta para presença de doença de Chagas e, consequiente megaesôfago chagásico. Neste caso, pela história clínica encontrada no prontuário do doente, foram estabelecidos os prováveis locais de contaminação pelo Trypanosoma cruzi, no intuito de se esboçar um perfil da doença, dos atendidos na UNICAMP.

\section{RESULTADOS}

Os pacientes operados de megaesôfago no Hospital de Clínicas da UNICAMP de 1987 a 2005 apresentaram o seguinte perfil: 216 (55\%) homens e 174 (45\%) mulheres, com as respectivas médias de idade: 46,5 e 47,7 anos (variação: 13-91 anos). Havia 170 (79\%) homens chagásicos e 46 (21\%) portadores da acalásia idiopática. No grupo das mulheres, 139 (80\%) eram chagásicas e 33 (19\%) não chagásicas. Não houve diferenças relevantes entre homens e mulheres em quaisquer aspectos, o que possibilitou agrupá-los conjuntamente neste estudo.

Naturalidade e procedência dos pacientes $\left(n^{0}=385\right.$ : cinco pacientes foram retirados dessa análise por ausência desses dados em seus prontuários). Foram operados pacientes nascidos em 15 estados diferentes, com a seguinte distribuição: 148 naturais do Estado de São Paulo, 112 naturais de Minas Gerais; 38 da Bahia; 26 do Paraná; 14 de Pernambuco; 14 de Alagoas; nove do Ceará; cinco da Paraíba; quatro do Piauí, três do Sergipe, três do Rio de Janeiro e um paciente natural de cada um dos seguintes Estados: Acre, Espírito Santo, Goiás, Rio Grande do Norte, Rondônia, Santa Catarina e Mato Grosso do Sul. Houve, ainda, um paciente de nacionalidade portuguesa e um de nacionalidade norte-americana, sem especificação de suas respectivas naturalidades. Assim, tem-se uma distribuição conforme ilustra a Figura 1.

Procedência. Os pacientes operados foram encaminhados ao Hospital de Clínicas por oito estados diferentes, embora sejam, esses pacientes, naturais de vários outros estados. Isso caracteriza a migração interna brasileira de anos, em especial para

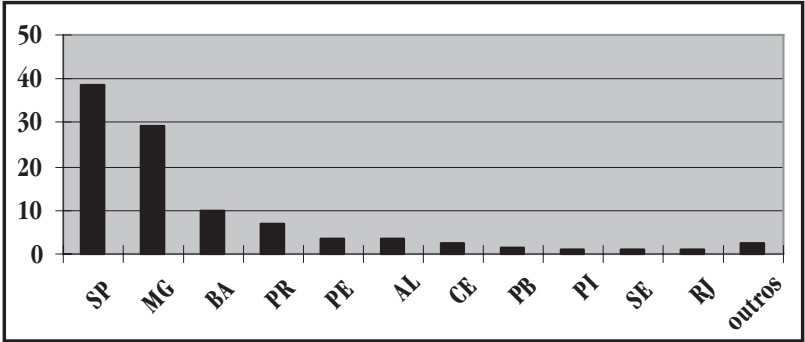

Figura 1 - Naturalidade dos pacientes e distribuição percentual, segundo unidades da federação.

o Estado de São Paulo, haja vista a grande parcela de pacientes procedentes ser deste estado. Esses dados também confirmam o papel do HC/UNICAMP de atender população do Estado de São Paulo e regiões adjacentes, em especial Minas Gerais e Paraná. Os pacientes foram distribuídos da seguinte forma: 348 procedentes de São Paulo, 26 de Minas Gerais, quatro do Paraná, três da Bahia e um paciente de cada um dos seguintes estados: Mato Grosso, Pará, Rondônia e Santa Catarina. A Figura 2 ilustra esses dados, em termos percentuais.

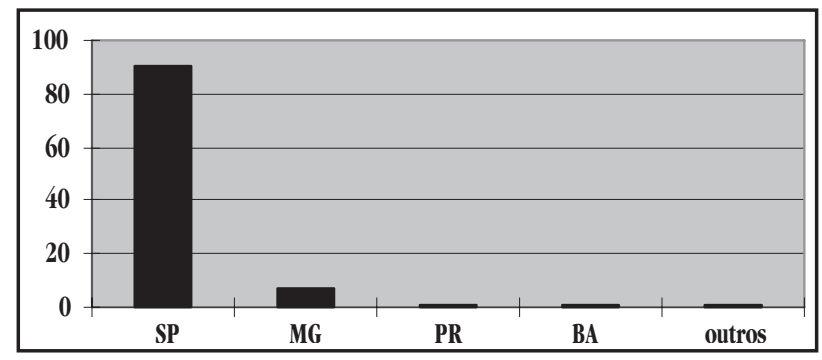

Figura 2 - Procedência dos pacientes e distribuição percentual, segundo unidades da federação.

Provável meio/local de contágio. Com os dados consultados nos prontuários dos pacientes chagásicos (naturalidade e procedência, histórias de migrações, contato com o barbeiro, transfusões de sangue, dentre outros), foi feita uma estimativa do provável meio/local de contágio em 242 casos de chagásicos (78\% de todos os contaminados). Três $(1,2 \%)$ pacientes se contaminaram, provavelmente, por meio de transfusão sangüínea, nas décadas de 60 e 70; eles negaram contato com barbeiro e dois destes nunca habitaram zona endêmica para Chagas. Os outros 239, provavelmente se contaminaram nos seguintes estados: 80 em Minas Gerais, 66 em São Paulo, 30 na Bahia, 26 no Paraná, 11 em Alagoas, oito em Pernambuco, seis no Ceará, três na Paraíba, três no Piauí, três no Sergipe e três em outros estados. Analisadas, especificamente, as cidades em que devem ter ocorrido esse contágio, obteve-se destaque para determinadas regiões: região norte de $\mathrm{MG}$, fronteira norte de $\mathrm{MG} / \mathrm{sul}$ da $\mathrm{BA}$, fronteira AL/PE, fronteira nordeste de SP/sul de MG, fronteira norte do PR/oeste de SP e região da Alta Mogiana (SP). As Figuras 3 e 4 ilustram a distribuição por estado e a localização dos casos bem delimitada no mapa brasileiro.

Duração da disfagia. Ao avaliar a disfagia, obtevese informações relevantes. Em 84,4\% dos casos, ela foi declaradamente progressiva, inclusive no caso dos pacientes 


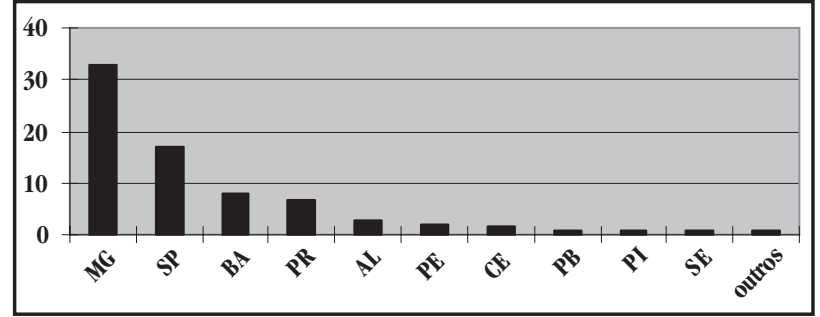

Figura 3 - Provável local de contágio dos pacientes e distribuição percentual, segundo unidades da federação.

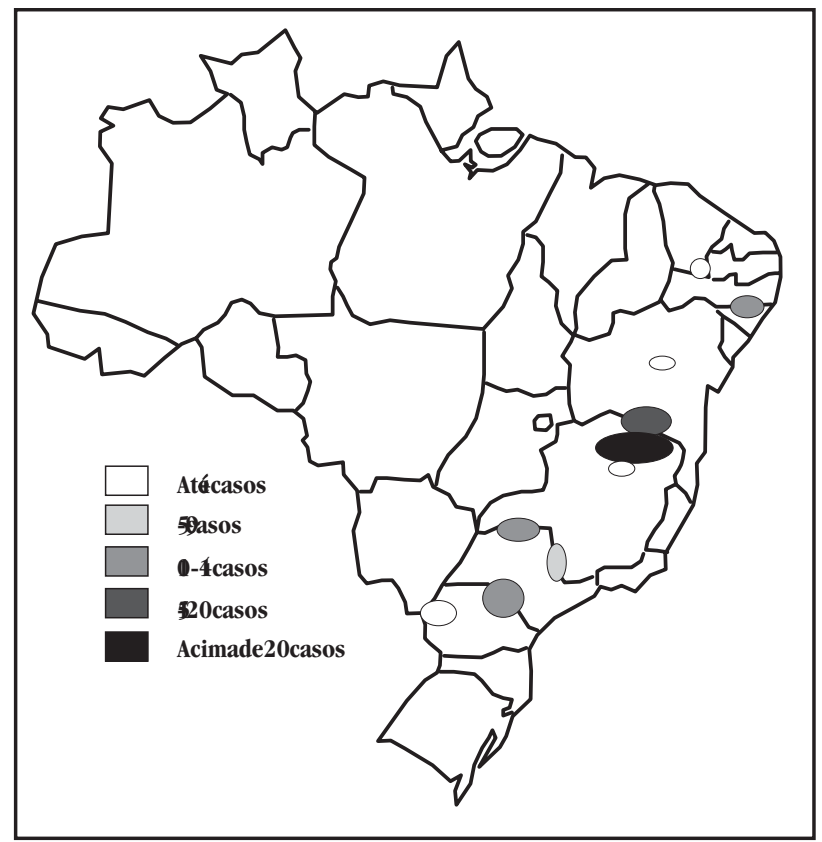

Figura 4 - Perfil epidemiológico com os prováveis locais de contágio dos pacientes chagásicos operados de megaesôfago no Hospital de Clínicas da Universidade de Campinas (1989-2005).

portadores de acalásia idiopática, o que revela o caráter progressivo de instalação da doença. A média foi de 9,47 anos até a procura de apoio médico. 0 período mais freqüentemente referido foi de dez anos de duração, com variação entre 15 dias a 60 anos de queixa. Destaca-se o fato de uma disfagia presente há dez anos ou mais ter forte indício de ser por doença chagásica, pois dos 121 casos observados, 108 (89\%) eram chagásicos.

Idade dos pacientes na data da cirurgia. A média de idade dos pacientes foi de 47 anos $\left(n^{0}=385\right)$, com um mínimo de 13 e um máximo de 91 anos. A distribuição por faixa etária (0 a 20 anos, 21 a 40 anos, 41 a 60 anos e acima de 60 anos) mostra a maior incidência de casos operados com idade de 40 a 60 anos, sendo menos freqüientes as cirurgias em pacientes com menos de 40 anos (37,5\% do total), conforme mostram as Figuras 5 e 6 .

Associação de outras doenças. Outras doenças no trato gastrintestinal foram observadas em 292 (76\%) doentes. As mais prevalentes foram: esofagite $(36,6 \%)$, gastrite $(54,4 \%)$, duodenite $(12,8 \%)$, megacólon $(18,4 \%)$ e colecistopatia calculosa $(10,4 \%)$.

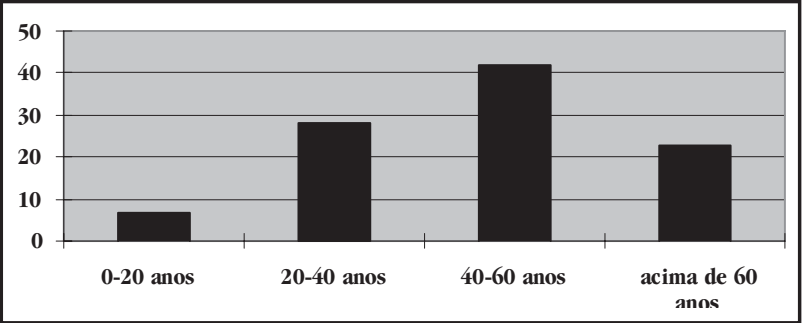

Figura 5 - Faixa etária: idade na data da cirurgia.

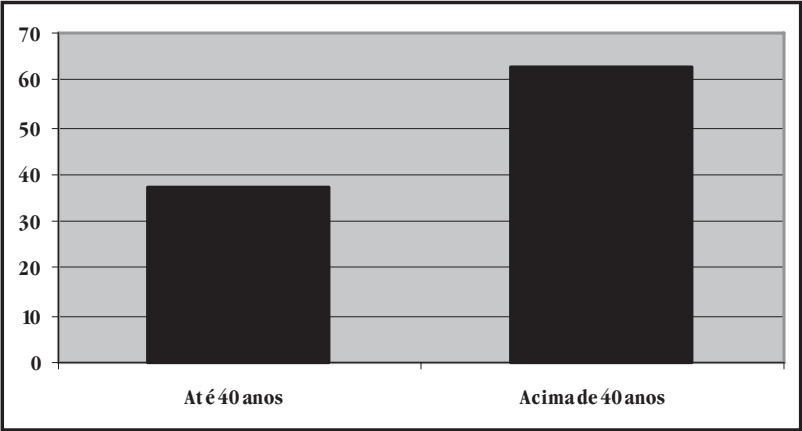

Figura 6 - Idade na data da cirurgia (\%).

Além dessas moléstias no TGI, houve outras duas doenças com prevalência relevante: hipertensão arterial sistêmica $(24,6 \%)$ e a cardiopatia (27,4\%). A Figura 7 permite visualizar essa associação de forma clara.

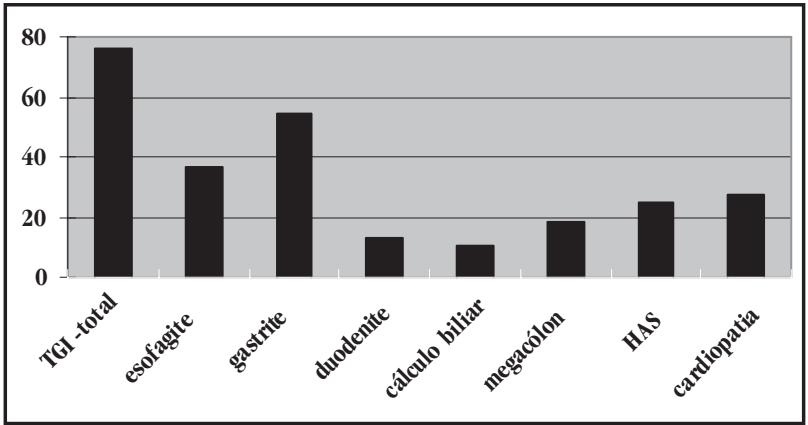

Figura 7 - Outras doenças associadas (\%).

Houve outras alterações anotadas, mas pouco prevalentes, como o divertículo de esôfago e duodenal, a hérnia de hiato paraesofágica, a hepato e a esplenomegalia, o diabetes melitus, além de pacientes com doenças psiquiátricas.

Grau do megaesôfago. Distribuição dos pacientes, segundo o grau do megaesôfago. Grau 1: 77 (20\%) casos. Grau 2: 189 (48\%) casos. Grau 3: 90 (23\%) casos. Grau 4: 38 (10\%) casos.

Modalidade cirúrgica adotada. Para os graus 1, 2 e 3, foi realizada a cirurgia de Heller-Pinotti, sendo, pois, a de maior escolha. Oito pacientes de grau 4 também foram submetidos à cirurgia de Heller, em razão de suas condições clínicas serem desfavoráveis. (acima de 70 anos e/ou com problemas cardio-respiratórios que ofereciam grande risco a cirurgias de maior porte).

0 total de cirurgias de Heller foi 361 (inclusive as remiotomias). No grau 4, à exceção dos oito pacientes citados, foi 
realizada esofagectomia (23 casos) ou mucosectomia (11 casos). As Figuras 8 e 9 mostram a porcentagem de pacientes, segundo o grau da doença e conforme a cirurgia de escolha.

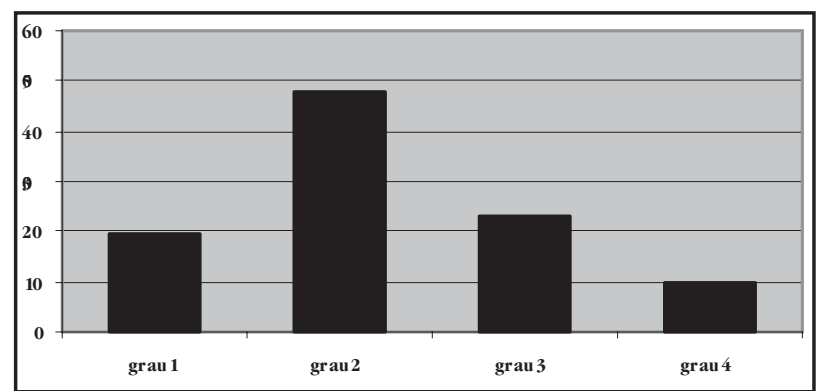

Figura 8 - Grau do megaesôfago (\%).

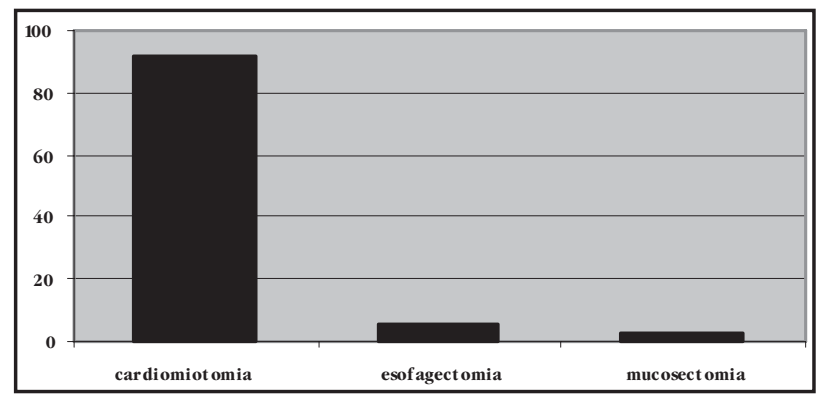

Figura 9 - Modalidade cirúrgica adotada (\%).

\section{DISCUSSÃO}

A análise dos resultados deste trabalho permite delimitar o perfil dos pacientes operados por megaesôfago no HC/ UNICAMP.

Com respeito à naturalidade, encontrou-se uma grande variedade de localidades: os pacientes eram naturais de quinze estados diferentes, além de haver dois estrangeiros. Essa variedade evidencia a migração interna brasileira, em especial para o Estado de São Paulo, ocorrida décadas atrás. Devido à moléstia de Chagas estar presente em 79\% dos doentes, a maioria advém de três estados em que, sabidamente, houve diversas zonas endêmicas: São Paulo, Minas Gerais e Bahia. Conforme mostra o mapa (Figura 4), as principais regiões de contágio dos chagásicos atendidos foram: norte de Minas Gerais, fronteira norte de Minas Gerais/sul da Bahia, fronteira Alagoas/Pernambuco, fronteira nordeste de São Paulo/sul de Minas Gerais, fronteira norte do Paraná/oeste de São Paulo e região da Alta Mogiana (SP). Já quanto à procedência dos pacientes, verificou-se um total de oito estados, sendo que $90 \%$ provêm do Estado de São Paulo, especialmente Campinas e regiões adjacentes. Esse dado caracteriza o papel do HC/UNICAMP como serviço referência para São Paulo e até alguns Estados próximos, como Minas Gerais e Paraná.

Avaliando-se a disfagia, conclui-se que ela é de instalação progressiva $(84,4 \%$ dos casos) e independe da etiologia do megaesôfago. Em média, durou 9,47 anos e foi mais freqüente a referência de dez anos de duração. Valores semelhantes são encontrados na literatura (entre 7 e 11 anos de duração) 23910. Destaca-se, como suspeita diagnóstica, que $89 \%$ dos casos com disfagia presente há dez anos ou mais, foram de pacientes chagásicos.

Sobre a idade dos pacientes, na data da cirurgia, 62,5\% tinham 40 anos ou mais; a faixa etária predominante foi da população adulta de 40 a 60 anos (43\% do total). Outros estudos também referem a predominância dessa faixa etária nos portadores de megaesôfago ${ }^{239}$. Em relação à associação de outras doenças, notou-se que o trato gastro-intestinal estava também acometido por outras doenças em $76 \%$ dos casos, sendo mais freqüentes: esofagite $(36,6 \%)$, gastrite $(54,4 \%)$, duodenite $(12,8 \%)$, megacólon $(18,4 \%)$ e colecistopatia calculosa $(10,4 \%)$. o impedimento da deglutição, nos doentes, deve ter relação com as inflamações apresentadas. Já o megacólon relaciona-se, diretamente à doença de Chagas. Outras duas doenças muito presentes foram a cardiopatia $(27,4 \%)$, normalmente, conseqüente à doença de Chagas (95\% destes eram também chagásicos) e a hipertensão arterial sistêmica $(24,6 \%)$.

A distribuição dos pacientes, segundo o grau do megaesôfago, mostra que as formas mais graves estão cada vez mais escassas, com prevalência dos graus 2, 3 e 1 (48\%, 23\% e 20\%, respectivamente). A evolução de medidas sanitárias, inclusive a borrifação de inseticidas e a informação da população sobre medidas que evitam a contaminação pelo barbeiro reduziram a incidência geral da doença no país. Tais medidas diminuíram também a ocorrência das doenças diretamente associadas, como o megaesôfago.

Medidas como: informar a população, construir centros de saúdes e realizar testes mais avançados para detectar essa doença (imunofluorescência, ELISA, além do tradicional Machado Guerreiro) facilitaram o diagnóstico e isso parece explicar a baixa prevalência da forma avançada na UNICAMP (10\%).

Outros autores também observaram essa menor prevalência ${ }^{3} 1013$. A conseqüência disso é a opção maior pela cirurgia de Heller-Pinotti, ocorrida em 92\% dos casos, esta mais simples e segura, com morbidade bem menor às outras opções (esofagectomia e mucosectomia), necessárias nos casos avançados.

Assim, de maneira geral, pode-se classificar o paciente atendido no HC/UNICAMP como: com idade entre 40 e 60 anos, chagásico, natural de provável zona endêmica, procedente do Estado de São Paulo, grau 2 da doença, com cerca de dez anos de queixas disfágicas, com alguma outra doença digestiva associada e candidato à cirurgia de Heller-Pinotti como forma terapêutica.

\section{AGRADECIMENTOS}

Os autores do trabalho agradecem aos membros da equipe de cirurgia do Aparelho Digestivo da Universidade de Campinas, aos funcionários do Serviço de Arquivo Médico do Hospital das Clínicas da Universidade de Campinas e aos pacientes cujos prontuários constituíram a fonte de pesquisa deste estudo. 


\section{REFERÊNCIAS}

1. Andreollo NA, Lopes LR, Brandalise NA, Leonardi LS. Acalasia idiopática do esôfago: análise de 25 casos. Gastroenterologia e Endoscopia Digestiva 15: 151-155, 1996

2. Carrilho RP. Estudo longitudinal de 25 anos da doença de Chagas em Mambaí/ Buritinópolis (GO) - Brasil. Tese de Doutorado, Universidade de Brasília (UnB), Brasília, DF, 2001.

3. Ceneviva R, Fereira-Santos R, Santos JS, Mente ED, Sankarankutty AK. Alterações cronológicas do perfil dos pacientes e da modalidade de tratamento cirúrgico do megaesôfago chagásico. Acta Cirurgica Brasileira 17 (supl III): 125-128, 2002.

4. Cirilo PM, Mantovani M. Temas de cirurgia do aparelho digestivo alto. Fundo Editorial Byk, São Paulo, SP, 2000.

5. Coelho JCU. Aparelho Digestivo - Clínica Cirúrgica. Editora Medsi, Rio de Janeiro, RJ, 1996.

6. Crema E, Cruvinel LAF, Werneck AM, Oliveira RM, Silva AA. Correlação manométrico-radiológica e sua importância no tratamento cirúrgico do megaesôfago chagásico. Revista da Sociedade Brasileira de Medicina Tropical 36: 665-669, 2003
7. Dantas R0. Idiopathic achalasia and Chagasic megaesophagus. Journal of Clinical Gastroenterology 10: 13-15, 1988

8. Dantas R0. Comparação entre acalásia idiopática e acalásia conseqüente à doença de Chagas: revisão. Arquivos de Gastroenterologia 40: 126-130, 2003.

9. Herbella FAM, del Grande JC, Lourenço LG, Mansur NS, Haddad CM. Resultados tardios da operaçäo de Heller associada à fundoplicatura no tratamento do megaesôfago: análise de 83 casos. Revista da Associação Médica Brasileira 45: 317-322, 1999 .

10. Herbella FAM, Oliveira DRCF, Del Grande JC. Are idiopathic and chagasic achalasia two different Diseases? Digestive Diseases and Sciences 49: 353-360, 2004.

11. Mascarenhas LG, Câmera-Lopes LH, Jurema B, Ferreira-Santos R. Padronização técnica da radiologia do megaesôfago. In: Anais do X Congresso Brasileiro de Gastroenterologia, Belo Horizonte, MG, 1958.

12. Park W, Vaezi MF. Etiology and pathogenesis of achalasia: the current understanding. American Journal of Gastroenterology 100: 1404-1414, 2005.

13. Silveira AC. 0 controle da doença de Chagas nos países do cone sul da América. História de uma iniciativa internacional - 1991/2001. Fundação de Ensino e Pesquisa de Uberaba, Uberaba, MG, 2002. 